\title{
SUBMAXIMAL OPERATOR SPACE STRUCTURES ON BANACH SPACES
}

\author{
VINOD KUMAR. P AND M. S. BALASUBRAMANI
}

Abstract. Subspaces of maximal operator spaces are called submaximal spaces and in general, they need not be maximal. We call those maximal operator spaces with the property that all submaximal spaces turn out to be maximal as hereditarily maximal spaces. Any two Banach isomorphic subspaces of a hereditarily maximal space will be completely isomorphic as operator spaces. We derive a characterization of these spaces. We introduce a notion of distance of an operator space to the class of submaximal spaces and discuss some related results.

Mathematics subject classification (2010): 46L07, 47L25.

Keywords and phrases: Operator spaces, maximal operator spaces, submaximal spaces, hereditarily maximal spaces.

\section{REFERENCES}

[1] D. Blecher, The standard dual of an operator space, Pacific J. Math. 153 (1992), 15-30.

[2] D. Blecher And V. PAulsen, Tensor products of operator spaces, J. Funct. Anal. 99 (1991), 262292.

[3] E. Effros And Z.-J. RuAn, Operator Spaces, London Math. Soc. Monographs. New Series, Oxford University Press, 2000.

[4] I.M. GELFAND AND M.A. NAIMARK, On the imbedding of normed rings into the rings of operators on a Hilbert space, Math. Sbornik. 12 (1943).

[5] T. OiknberG, Subspaces of Maximal Operator Spaces, Integr. equ. oper. theory 48 (2004), 81-102.

[6] V. PAUlsen, Representation of function algebras, Abstract operator spaces and Banach space geometry, J. Funct. Anal. 109 (1992), 113-129.

[7] V. PAUlsEn, The maximal operator space of a normed space, Proc. Edinburgh. Math. Soc. 39 (1996), 309-323.

[8] V. PAulsen, Completely Bounded maps and Operator Algebras, Cambridge University Press, 2002.

[9] G. PISIER, Introduction to operator space theory, Cambridge University Press, 2003.

[10] E. RICARD, A tensor norm for $\boldsymbol{Q}$-spaces, J. Operator theory 48 (2002), 431-445.

[11] Z.-J. RuAn, Subspaces of $\boldsymbol{C}^{*}$-algebras, J. Funct. Anal. 76 (1988), 217-230. 